\title{
Der Farngarten von Santiago de Cuba
}

\author{
Mónica Palacios-Rios, Manuel G. Caluff \& Gustavo Shelton
}

\begin{abstract}
The fern-garden of Santiago de Cuba owns the largest fern collection of the Antilles, comprising more than 300 species cultivated in 5 different areas, which are designed to accomplish their ecological needs. Besides the cautious cultivation and care of threatened and endemic species, it is devoted to the botanical research and teaching.
\end{abstract}

\section{Resumen}

El jardín de helechos de Santiago de Cuba posee la colección de pteridofitas más grande de las Antillas, comprendiendo más de 300 especies que se cultivan en 5 áreas diferentes para cumplir con sus necesidades ecológicas. Además del cultivo y cuidado de especies amenazadas y endémicas, se dedica a la investigación y enseńanza de botánica.

\section{Zusammenfassung}

Der Farngarten von Santiago de Cuba beherbergt die mit 300 Arten größte Farnsammlung der Antillen, die in fünf verschiedenen, speziell ihren ökologischen Standortansprüchen gerechten Bereichen kultiviert werden. Außer der behutsamen Anzucht und Pflege von bedrohten und endemischen Arten widmet er sich der botanischen Forschung und Lehre.

\section{Artenvielfalt und Forschung im Garten}

Die Stadt Santiago de Cuba, im südöstlichen Teil von Kuba gelegen, ist nach Havanna die zweitwichtigste Stadt des Landes. Der Farngarten liegt nahe der Stadt in attraktiver Lage am Fuße der herrlichen Gran-Piedra-Berge auf einer Höhe von $30 \mathrm{~m}$ über dem Meeresspiegel. Er beherbergt die umfangreichste Sammlung lebender Farnpflanzen der Antillen und widmet sich auch bedeutenden Forschungsaufgaben. Der Garten umfasst $2000 \mathrm{~m}^{2}$ und ist Heimstätte für ungefähr 3000 Pflanzenarten. Rund 300 dieser Arten sind Farne und Farnverwandte, die nach ihren spezifischen ökologischen Standortansprüchen im Garten verteilt sind. Von ihnen sind $85 \%$ auf Kuba heimisch. Aufgrund des geschlossenen Baumkronendaches und der anhaltend hohen Temperaturen schwankt die relative Luftfeuchtigkeit nur geringfügig zwischen 75 und $95 \%$. Die durchschnittliche Jahrestemperatur liegt bei $25^{\circ} \mathrm{C}$. Der Boden ist alluvialen Ursprungs und schwach sauer mit einem pH-Wert von 6,2.

Die Farnsammlung repräsentiert alle vier Pteridophytenklassen, 27 Familien und 61 Gattungen. Von den 300 Arten werden 16 als bedroht und 27 als selten eingestuft. Unter den Farnen befinden sich 11 Arten kubanischer En- demiten. Die Sammlung hat eine bedeutende Rolle in der kubanischen Farnforschung, besonders in den Bereichen der Taxonomie, der Anzucht und des Artenschutzes. Bedrohte Arten sollen in Zukunft auch wieder an ihren $\mathrm{Na}$ turstandorten eingeführt werden. Eine weitere Aufgabe des Botanischen Gartens besteht in der Lehre. Denn er ist die Zentralstelle für Botanik-Studenten aller Semester, die im Garten ihre praktischen Erfahrungen sammeln können. Eine vollständige Liste der im Garten kultivierten Farne und Farnverwandten kann bei Bedarf über die Erstautorin oder die Redaktion dieses Heftes bezogen werden.

Der Zweitautor dieses Beitrages ist Direktor des Botanischen Gartens und der Forschungsstätte. Er gründete den Garten 1976 mit den bescheidenen eigenen Pflanzensammlungen und unter Mithilfe seiner Familie und seines Kollegen, dem Drittautor dieses Berichtes. Heute gehört er zum „Centro Oriental de Biodiversidad de la Academia de Ciencias de Cuba."

Die Gartenverwaltung ist am Sporenaustausch mit anderen Farngesellschaften, Botanischen Gärten und Privatsammlern interessiert. Der Farngarten ist in fünf abgegrenzte Bereiche eingeteilt. 


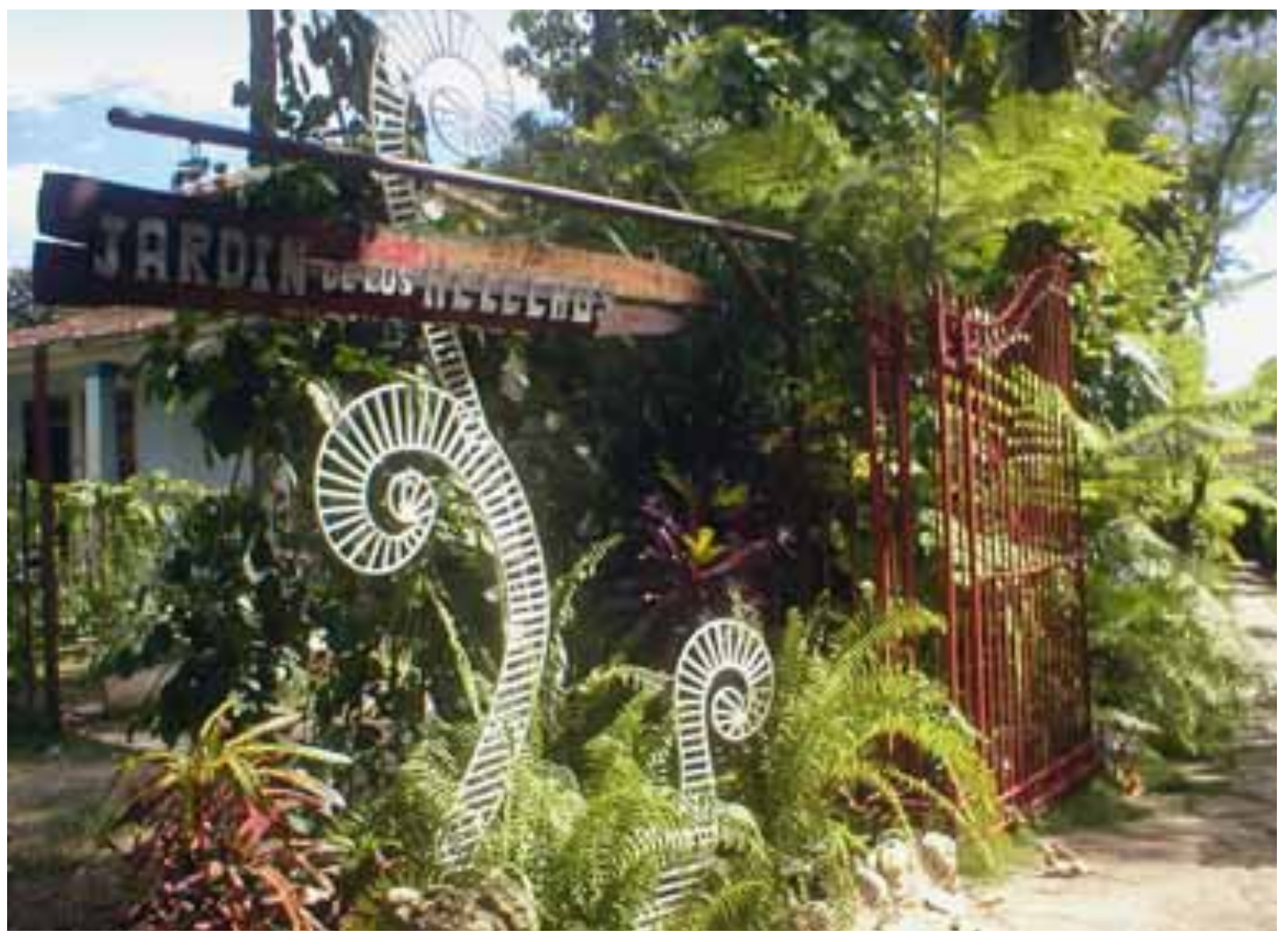

\section{Die verschiedenen Farnsammlungen des Gartens}

\subsection{Zierfarne}

In diesem Bereich des Gartens werden häufig als Haus- und Gartenpflanzen kultivierte Farnarten gezeigt. Hier befinden sich unter anderem sechs Varietäten von Nephrolepis exaltata und neun weitere Arten dieser Gattung. Besonders auffallend sind mehrere kubanische AdiantumArten (Frauenhaarfarne) und exotische Vertreter der Tüpfelfarne aus der Gattung Polypodium. Dieser Gartenbereich findet vor allem bei Farnliebhabern großen Anklang, die auf der Suche nach Namen und Heimat ihrer dekorativen Zierpflanzen sind.

\subsection{Felsfarne oder epilithische Farne}

An einer senkrechten Felswand werden die natürlichen Standortbedingungen der epilithischen Farne und Farnverwandten möglichst

Abb. 1: Garteneingang an der Hauptstraße von El Caney. gut nachgeahmt, indem das ständig von der Felswand tropfende Wasser auf ein künstliches Substrat fällt, welches über eine ausgezeichnete Drainage verfügt. Rund 150 Arten werden hier ausgestellt. Die meisten von ihnen sind auf Kuba heimische, kalkliebende Farne, die sich hauptsächlich auf vegetative Weise vermehren. Die artenreichsten Gattungen unter diesen sind Thelypteris, Tectaria, Adiantum und der Moosfarn Selaginella. Von besonderem Interesse ist die hier kultivierte Microlepia speluncae, eine auf Kuba sehr seltene Art.

\subsection{Halbschatten- und Schattenfarne}

Für Farne mit geringerem Lichtbedarf wurden spezielle Vorkehrungen getroffen. Ein Bereich von $300 \mathrm{~m}^{2}$ wurde mit der Kletterpflanze Thunbergia alata überdacht und ein zweiter Bereich wurde mit Blättern der Kokospalme abgedeckt. Letzterer schirmt die Lichteinstrahlung in geringerem Maße ein, so dass hier die Halbschattenfarne ihren Platz finden. An den Seiten grenzt ein natürlicher Vorhang aus Aralien den 


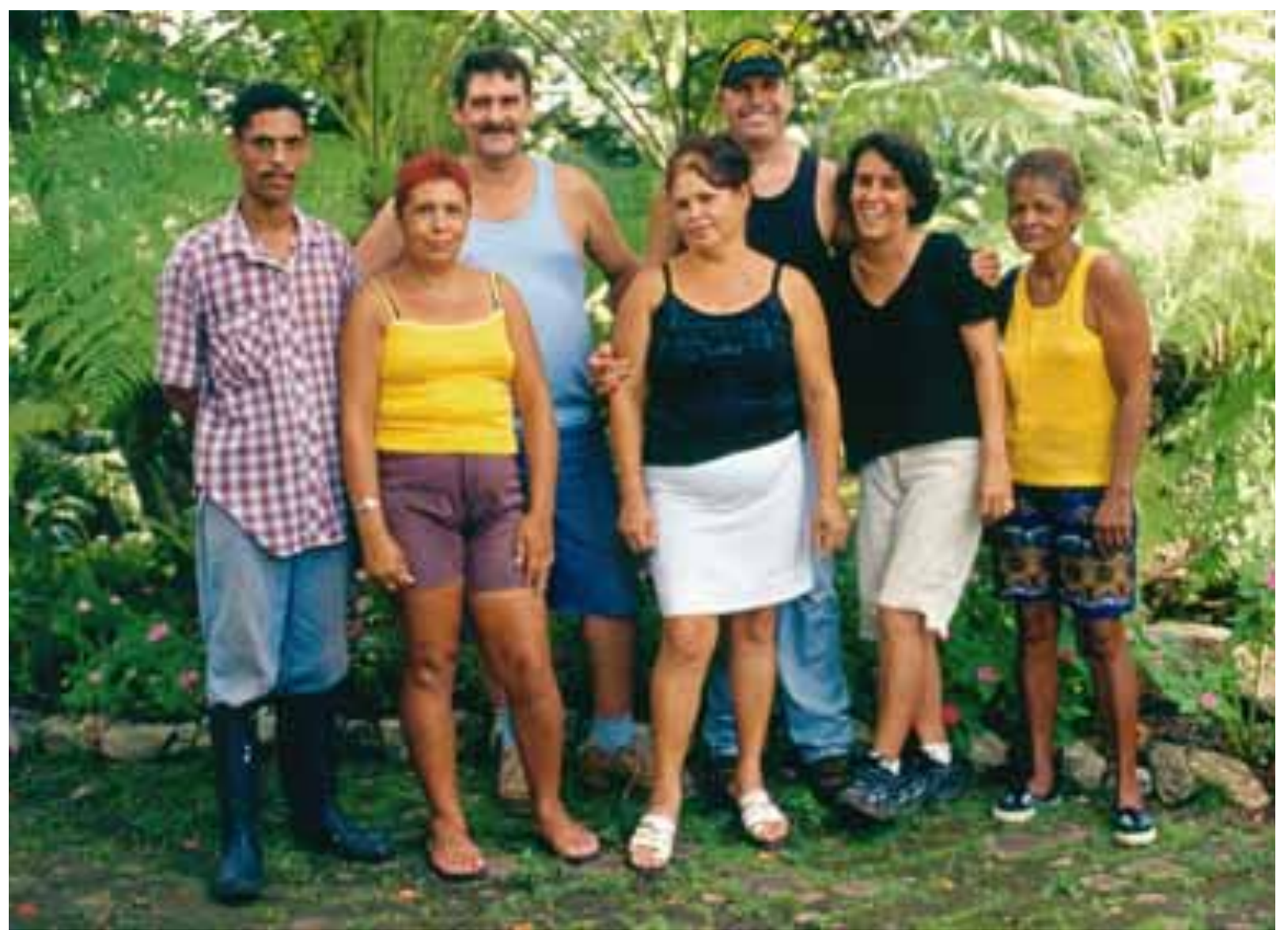

Schattenbereich ab. Die Schatten- und Halbschattenfarne erhalten eine ihren individuellen Ansprüchen gerechte Bodenmischung, wobei speziell darauf geachtet wird, ob es sich um acido-, basi- oder neutrophile Arten handelt.

\subsection{Sonnenfarne: Wasser- und Küstenfarne}

Die Mehrzahl der Wasser- und Küstenfarne werden in Tanks kultiviert. Jede Art erhält einen separaten Wasserbehälter, um Konkurrenzeinflüsse zwischen den Arten auszuschließen. Insbesondere aggressivere Arten der Wasserfarne neigen zu schnellem Wachstum und eindrucksvoller vegetativer Vermehrung, so dass sie leicht die Oberherrschaft über andere Arten gewinnen können.

\subsection{Baumfarne}

Eine der größten und meist bestaunten Attraktionen des Botanischen Gartens von Santiago de Cuba ist der Bereich der Baumfarne. Es ist beeindruckend, diese eleganten Pflanzen mit ihren über $3 \mathrm{~m}$ langen Blättern in einer Höhe von nur wenigen Metern über dem Meeresspiegel bewundern zu können, da es sich bei ihnen gewöhnlich um Bewohner der mittleren Gebirgslagen handelt. Aufgrund der besonderen Pflege entwickelt Cyathea arborea seit Jahren fertile Wedel. In mehreren Bereichen des Gartens gehen aus ihren Sporen sogar Jungpflanzen hervor. Zur Zeit können im Garten von den Baumfarnen 14 Arten und 2 Hybriden bewundert werden.

Aufgrund der sorgfältigen gärtnerischen Methoden vermehren sich im Garten rund 100 der 300 Farnarten erfolgreich auf generativem Wege. Statt Kunstdünger wird Naturkompost

Abb. 2: Mitarbeiterinnen und Mitarbeiter des Farngartens.

Abb. 3 (Seite 43 oben links): Doryopteris ludens.

Abb. 4 (Seite 43 oben rechts): Unterseite des Wedels von Doryopteris ludens mit Sporangien.

Abb. 5 (Seite 43 unten links): Pteris bahamensis.

Abb. 6 (Seite 43 unten rechts): Cyathea arborea. 

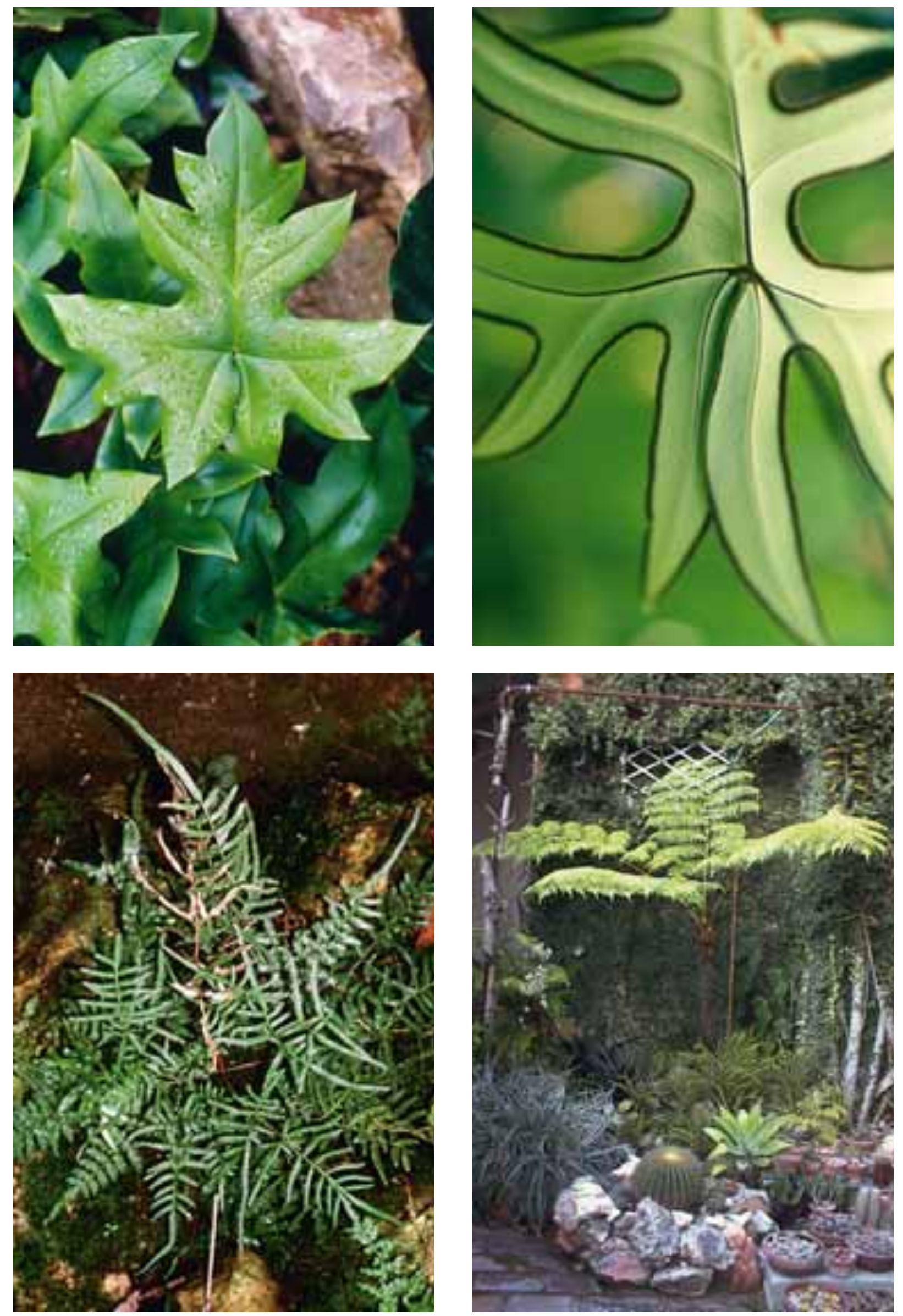

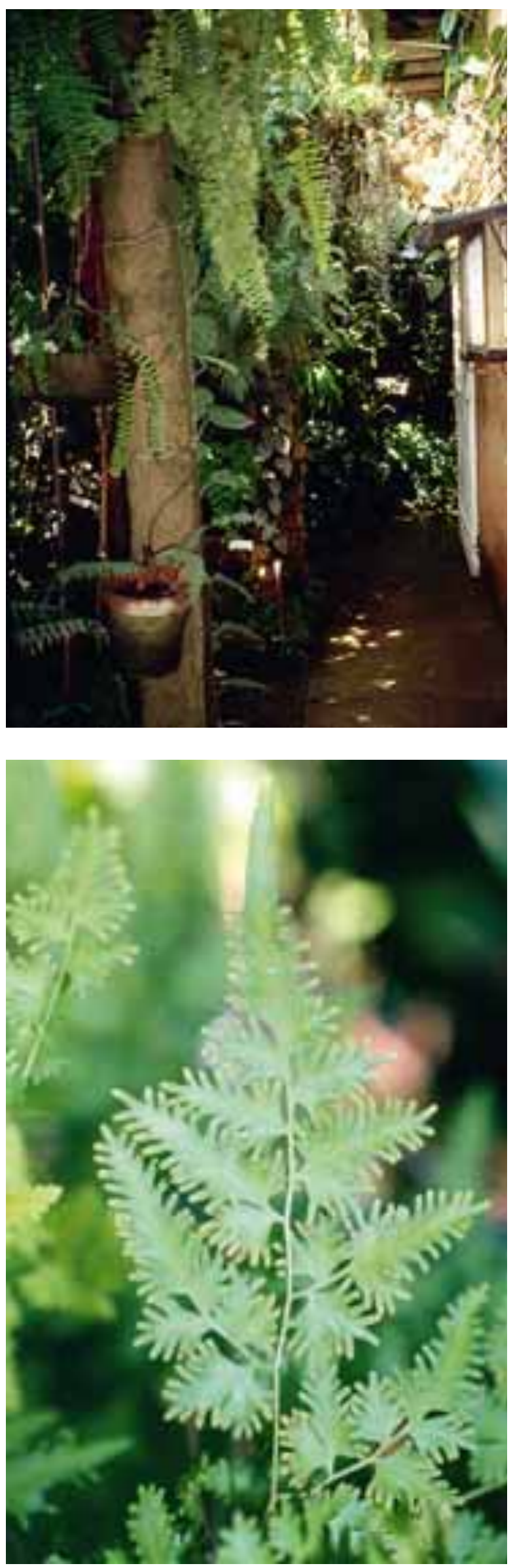

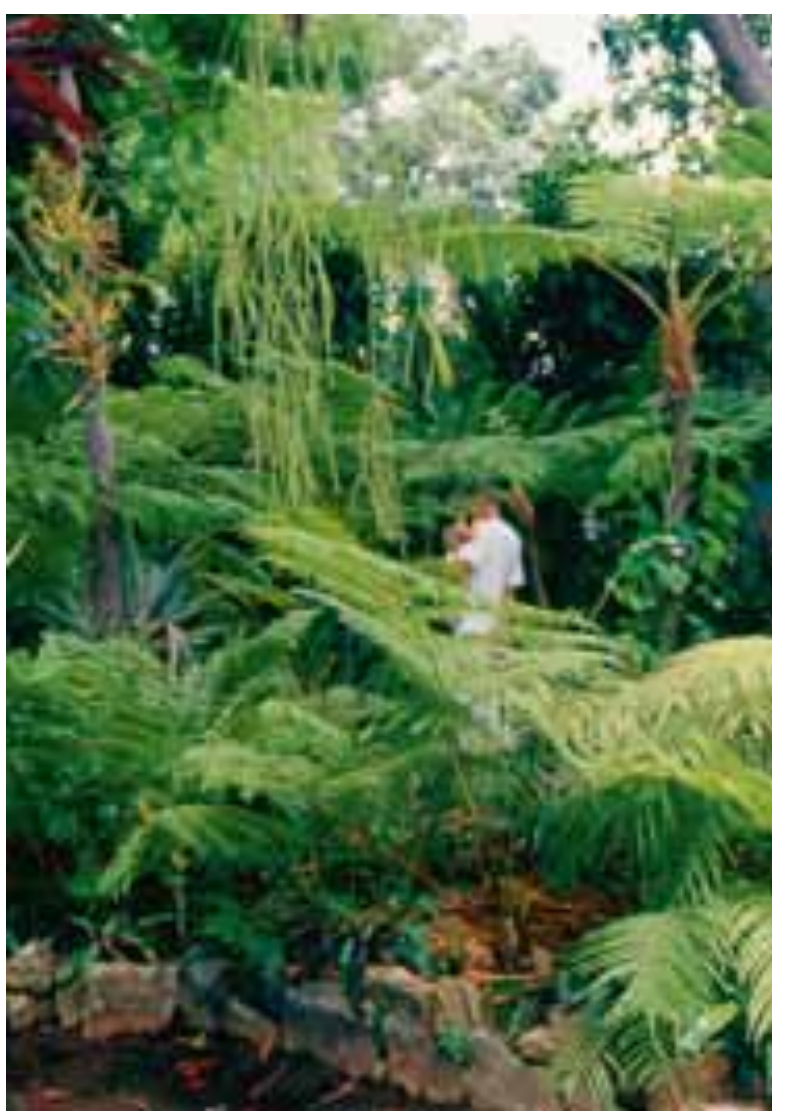

eingesetzt, der aus geschnitzelten Gartenabfällen gewonnen wird. Der bei der Verrottung resultierende Humus eignet sich ausgezeichnet für die Kultur von Farnen. Gehäuse- und Nacktschnecken werden durch biologische Schädlingsbekämpfung, in diesem Falle durch Frösche, kontrolliert.

\section{Literatur}

Shelton, G. \& Caluff, M. G. 1994: Helechos y plantas afines (Pteridophyta) que se reproducen espontáneamente en el Jardín de Helechos de la Academia de Ciencias de Cuba, Santiago de Cuba. Centro Oriental de Biodiversidad. Santiago de Cuba. (Unveröffentlichtes Manuskript)

\section{Internetseite}

http://www.helechos.com.mx/3Proyectos/3Jardin_Botanico_de_Santiago_de_Cuba/3cJardin_de_Helechos_de_ Santiago_de_Cuba_aleman/3cJardin_de_Helechos_de_ Santiago_de_Cuba_aleman.html

Abb. 7 (oben links): Bereich, in dem es zur Hauptsammlung des Gartens geht.

Abb. 8 (oben rechts): Im „Farndschungel“.

Abb. 9 (unten): Lygodium venustum. 